\title{
Propagation of radical ideas in societies with memory: competition between radical strength and social cohesion
}

\author{
Andrés Vallejo, Arturo C. Marti \\ Instituto de Física, Universidad de la República, Montevideo, Uruguay
}

\begin{abstract}
Based on a dynamical model with fractional derivatives we show that the evolution of radical groups in a society with a memory depends mainly on the active proselytism of radical groups and the level of cohesion in social networks. We find the conditions that determine that the radical group either disappears or, on the contrary, achieves a permanent significant expression. We also discuss the possible intervention strategies on the susceptible population and show the conditions that lead to the disappearance of radical groups. We see that the higher the level of memory, the maximum proportion of radicals decreases, but those groups manage to maintain their presence in society during a larger period of time.

Keywords: radical ideas, fractional calculus, memory, compartmental models
\end{abstract}

\section{Introduction}

Since the appearance of the well-known SIR (susceptible-infected-recovered/ removed) model for the spread of epidemics proposed by Kermack and McKendrick in 1927 [1, compartmental models have become so popular that they are now, as a first approximation, the standard approach used in the quantitative study of the spread of infectious diseases [2]. The basic idea of this kind of model is to divide the relevant population into a set of classes or compartments, which are defined according to the characteristics of the disease to be studied, and then to model the population flows between these compartments by means of a set of equations describing the dynamics of the system. The results of the analytical and numerical studies are interpreted in epidemiological terms for the purpose of predicting the evolution of infections and, given the case, defining epidemic control mechanisms. As these models became popular, it was clear that their mathematical structure transcended the field of epidemiology and that they were suitable for describing other phenomena in the social and human sciences. As a consequence, compartmental models have been used to study, with notable success, processes as varied as the acceptance of new scien- 
tific ideas [3, 4], language competition [5], the propagation of memes and rumors in social networks [6], or the dynamics of addictions [7.

In line with this approach, we propose a simple compartmental model to describe the spread of radical ideas in a society. While the mere existence of radical views does not necessarily pose a real problem, some extreme cases such as terrorist groups, dangerous sects or political extremists are a cause for concern in today's societies. For this reason, the problem of radicalization has been extensively addressed by the social sciences and, more recently, from interdisciplinary perspectives including mathematical modeling [8, 9, 10, 11, 12, 13. In this work, we rely on the analogy between, on the one hand, the spread of an epidemic whose contagion occurs by direct contact between infected individuals and susceptible individuals, and on the other hand, the processes of radicalization considered in a simplified way as the result of social interaction between individuals who already profess and proselytize the radical ideology (the "infected"), and individuals who are susceptible to adopt such ideology.

Compartmental models are usually based on a Markovian approximation where the future evolution of the system depends only on the state of the system at a single point in time. In many social systems, this hypothesis is adequate as a first approximation; however, we cannot ignore that, in these phenomena, memory (i.e. the history of the system in a certain time interval) plays an important role. A natural way to introduce memory into modeling is by using fractional calculus [14. Unlike ordinary derivatives, derivatives of non-integer order also depend on the previous evolution of the system, which makes a strong case for modeling systems with memory. Since this was first observed, fractional models have been used progressively in the study of this type of systems, reaching in many cases a level of accuracy in the description much higher than models based on standard calculus 15, 16. In this article, we consider the effects of the memory in our compartmental model of the propagation of radical ideas in societies.

In the next section we present the hypotheses of the model and arrive at the system of equations that governs its dynamics under the Markovian approximation. After that, in Section 3 we present the analytical and numerical study, obtaining and classifying the equilibrium points, qualitatively showing the possible evolutions of the system, and interpreting the results. In Section 4 , we introduce the standard procedure that allows the inclusion of memory in dynamical systems by means of Caputo's derivatives. These ideas are applied to our model in Section 5 where memory effects in the radicalization process are discussed. Finally, Section 5 is devoted to the conclusion.

\section{The model}

Conceptualizing radicalization is a difficult task, on which there is no consensus among experts. There is some agreement that it is a gradual process, a product of socialization, by which individuals or groups adopt an extremist worldview not shared by the mainstream of society, and tend to justify the use of radical mechanisms (such as the use of violence) to produce the desired 
social change [17]. Experts also differentiate between cognitive radicalization, which occurs at an ideological level and whose characteristics are those described above, and behavioral radicalization, which can range from moderate, public and legal activism in favor of the cause (for example, in social networks), to explicit violent action [17. These extreme cases constitute what is called violent radicalization, a process that generally includes the use of specific mechanisms such as selective recruitment and training. These characteristics, added to the fact that the number of individuals involved in such actions is usually marginal, make it difficult or directly question the relevance of a quantitative analysis of the phenomenon [18. On the other hand, studies suggest that cognitive and moderate behavioral radicalization are often a necessary condition for the emergence of social violence 19, which is why we will focus on understanding these processes.

It is therefore reasonable to propose compartmental models for the study of radicalization. Adapting the terminology of the SIR model, the population susceptible to embrace the radical idea is termed $S$, the population affiliated to that radical ideology and seeking to disseminate it is termed $R$, and $I$ the $\mathrm{im}$ munized individuals who either left the radical group thus acquiring immunity, at least transitory, or who are not very prone to adopt that position (moderates, who may be present even in the absence of radicals). Disregarding demographic changes due to births, deaths and migratory flows, the sum of the populations of the three compartments will be a constant, equal to the total population $N$ :

$$
S+R+I=N
$$

Following the philosophy adopted in compartmental models, we establish that all flows resulting from the interaction between individuals in different compartments are assumed to be proportional to the product of the populations. This assumption, usually adopted as a first approximation, is known as the law of mass action and implies uniform mixing of the populations [20]. In addition, we will take all model parameters (spontaneous transition rates, average number of contacts per unit time) as constants.

To model the dynamics of radical groups, we establish a set of specific hypotheses about the phenomenon that exhibit clear differences with traditional SIR models:

- Radical individuals actively proselytize to convince those likely to join their group. The level of militancy of each radical individual is represented by a parameter $\alpha$. We assume that each radical has $\alpha$ effective contacts per unit time, $S / N$ of which occur with susceptible individuals. Thus, $\alpha R S / N$ individuals will leave the group $S$ per unit of time.

- The radical nature of the idea being propagated means that the above interactions may result not only in conviction, but also in active rejection. We will model this rejection by assuming that only a fraction $p$ of those who left the susceptible group by interaction with the radicals adhere to the group $R$, while the rest, by opposition, pass directly to the compartment $I$, without having been radicalized. The parameter $p$ will depend 
on the efficiency of the social strategies employed, as well as on cultural factors.

- The immunized population can intervene to prevent radicalization (or in the absence of radicalization, to combat high levels of susceptibility), which we will model as an interaction with susceptible individuals at a rate $\beta$ of effective contacts per immunized individual, per unit of time (in this case we will assume that the effect of this intervention can only be null or positive). Consequently, $\beta S I / N$ individuals per unit time will transit from the compartment $S$ to the compartment $I$. The parameter $\beta$ will be an indicator of the intensity of the actions on the vulnerable population, and, more abstractly, of the levels of activism and social solidarity.

- Since radicalization typically arises out of opposition to a status quo that one wishes to combat [21, we will assume that the interaction between radicalized and immunized individuals has a negligible effect in terms of the flow between the compartments. On the contrary, it is often observed that interventions seeking to disrupt radicalization lead to a strengthening of the sense of belonging among the members of the radical group [22]. In more extreme cases, the interaction with immunized individuals may be prevented for security reasons (as in the case of terrorist groups) or even outright prohibited (as in the case of some dangerous sects) [23, 22, 24, 25].

- Different reasons, loss of faith in the ideology, rejection of the strategies employed, desire to return to a normal life, can produce the decision to spontaneously leave the radical group. So we assume a constant recovery rate $\gamma$ which depends on the level of contentment provided by belonging to it, as well as on the group's ability to retain its members. This will produce a $\gamma I$ flow of individuals per unit time that will pass from compartment $R$ to compartment $I$.

- Similarly, immunized individuals may, under certain conditions, become susceptible to radicalization. We will also assume that this occurs proportionally to the immunized population at a rate $\delta$, which, like $\gamma$, is the inverse of the average time of belonging to the group. According to this hypothesis, individuals per unit of time will move from compartment $I$ to compartment $S$. Except for personal factors, $\delta$ may be linked to the level of social welfare and may increase in situations of economic or social crisis.

Under the Markovian approximation, the above hypotheses result in the following system of differential equations:

$$
\left\{\begin{array}{l}
\frac{d s}{d t}=-\alpha s r-\beta s i+\delta i \\
\frac{d r}{d t}=p \alpha s r-\gamma r \\
\frac{d i}{d t}=\gamma r+\beta s i-\delta i+(1-p) \alpha s r
\end{array}\right.
$$




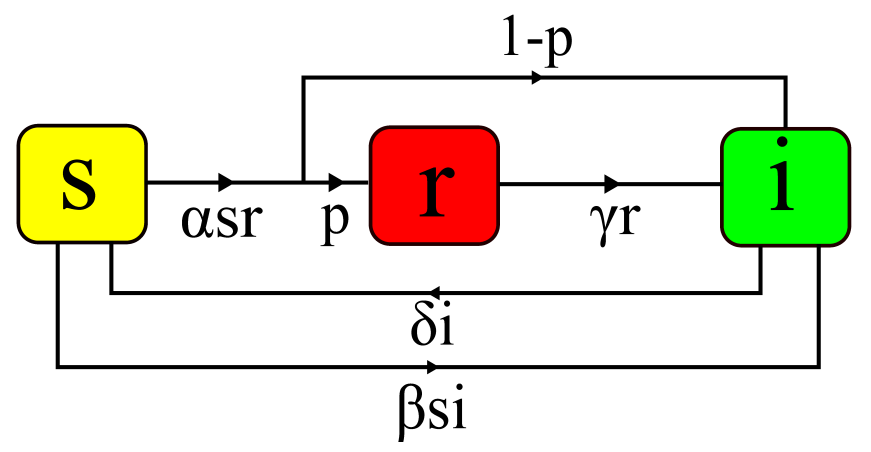

Figure 1: A schematic representation of the interactions between the populations.

where $s, r$ and $i$ and denote the fractions of the total population in each of the compartments at the point in time $t$ and verify $s+r+i=1$. An schematic representation of the interactions between the population is given in Fig. 1 .

\section{Analysis in the Markovian model}

\subsection{Equilibrium points}

From Eq2 we easily obtain the equilibrium points of the system:

$$
\begin{aligned}
& P_{1}=(1,0,0), \\
& P_{2}=\left(\frac{\delta}{\beta}, 0,1-\frac{\delta}{\beta}\right), \\
& P_{3}=\left(\frac{\gamma}{p \alpha}, \frac{(p \alpha \delta-\beta \gamma)(p \alpha-\gamma)}{p \gamma(p \alpha \delta-\beta \gamma+\alpha \gamma)}, \frac{\gamma(p \alpha-\gamma)}{p(p \alpha \delta-\beta \gamma+\alpha \gamma)}\right) .
\end{aligned}
$$

We verify that $P_{1}$ is an equilibrium point for any set of parameter values (which corresponds to the disease-free equilibrium of epidemiological models [2]). Given that $s, r, i \leq 1, P_{2}$ is also an equilibrium point only if $\delta \leq \beta$. As for $P_{3}$, we deduce the necessary condition of existence, $\gamma \leq p \alpha$, by observing its first component $(\mathrm{C} 1)$. Then, from the non-negativity of the third component, we obtain $p \alpha \delta-\beta \gamma+\alpha \gamma>0(\mathrm{C} 2)$. This result, together with the non-negativity of the second component leads to the additional condition $p \alpha \delta-\beta \gamma>0$ (C3). Given that $\alpha \gamma \geq 0$, it is evident that condition (C3) implies (C2). In summary:

$$
\begin{aligned}
& \exists P_{1} \forall \alpha, \beta, \gamma, \delta, p \\
& \exists P_{2} \leftrightarrow \delta \leq \beta \\
& \exists P_{3} \leftrightarrow \gamma \leq p \alpha \wedge p \alpha \delta \geq \beta \gamma
\end{aligned}
$$

The analysis of the above conditions shows that it is possible to significantly simplify the problem, reducing the number of parameters from five to two by 
defining the following:

$$
\Omega=\frac{\beta}{\delta} ; \quad \Lambda=\frac{p \alpha}{\gamma} .
$$

In terms of the new parameters, the conditions of existence, Eq. (4), are expressed as

$$
\begin{aligned}
& \exists P_{1} \forall \Omega, \Lambda \\
& \exists P_{2} \leftrightarrow \Omega \geq 1 \\
& \exists P_{3} \leftrightarrow \Lambda \geq 1 \wedge \Lambda \geq \Omega \leftrightarrow \Lambda \geq \max (1, \Omega)
\end{aligned}
$$

Parameters $\Lambda$ and $\Omega$ fully characterize the long term dynamics of the system. $\Lambda$ is the average number of susceptible individuals recruited by each radicalized individual while remaining in the group and we will refer to it as radical strength because it is a measure of the recruitment and retention capability of the radical group. This parameter is analogous to the basic reproduction number in epidemiological models [20. Similarly, $\Omega$ represents the expected value of the number of individuals that each immunized individual managed to remove from the susceptible state. A society will present a high value of $\Omega$ if, in parallel to reasonable levels of welfare that decrease the probability of becoming susceptible (small $\delta$ ), there is an important level of positive interaction among its members (large $\beta$ ), so it can be considered an indicator of social cohesion [26, 27.

Regarding the initial states that we will consider, in the absence of radical groups the dynamics described by Eqs. 2 is equivalent to that of the model $S I S$ for the spread of diseases that do not provide immunity [2]. The initial state considered will consist of a small fraction of radicalized individuals for the purpose of studying their evolution.

\subsection{Stability analysis and interpretation of results}

From the conditions (6) we can show that there are five regions in the parameter space $(\Lambda, \Omega)$ (see Fig. 7 below), characterized by the existence of the different possible combinations of equilibrium points. In order to simplify the stability study of the system (2), we use the conservation of the total population and take as variables the radicalized and the immunized, resulting in the following:

$$
\left\{\begin{aligned}
\frac{d r}{d t}= & (p \alpha-\gamma) r-p \alpha r i-p \alpha r^{2} \\
\frac{d i}{d t}= & (\gamma+(1-p) \alpha) r+(\beta-\delta) i-\beta i^{2} \\
& -(\beta+(1-p) \alpha) r i-(1-p) \alpha r^{2}
\end{aligned}\right.
$$

from which the Jacobian matrix can be easily obtained.

\subsubsection{Case 1: $\Omega<1 \wedge \Lambda<1$}

Given that $\Omega<1$, society is in the susceptible state prior to the appearance of the radical group the, and according to the discussion (6), under these conditions 
the only equilibrium point of the system (2) is $P_{1}=(1,0,0)$. The eigenvalues of the Jacobian matrix at that point are the following:

$$
\left\{\begin{array}{l}
\lambda_{1}^{P_{1}}=p \alpha-\gamma=\gamma(\Lambda-1)<0 \\
\lambda_{2}^{P_{1}}=\beta-\delta=\delta(\Omega-1)<0
\end{array}\right.
$$

Therefore, it will be asymptotically stable. Should a small radical group appear (regardless of size), it will disintegrate and the population will quickly return to the susceptible state.

This situation corresponds to societies with low social cohesion $(\Omega<1)$, but in which the radical group does not manage to develop a good capacity for attracting susceptible individuals or retaining its current members $(\Lambda<1)$. Figure 2 shows the temporal evolution of the populations of the three compartments for a somewhat artificial case in which $10 \%$ of the population becomes radicalized, showing an initial exponential decay in this group. This can be understood by noting that the equation governing that compartment at the beginning of the evolution is, approximately:

$$
\frac{d r}{d t}=\left(p \alpha s_{0}-\gamma\right) r=\gamma\left(\Lambda s_{0}-1\right) r
$$

and under the condition $\Lambda<1$, the first factor is negative for any initial fraction of susceptible individuals $s_{0}<1$. That decay produces a flow towards $I$, a group that is quickly abandoned due to the low levels of cohesion.

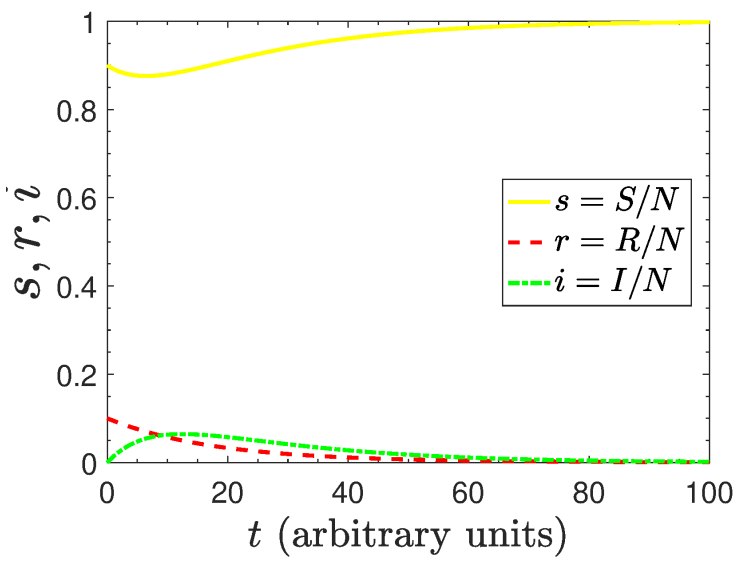

Figure 2: Evolution of the populations in case 1: in a society with low social cohesion and poor capacity of the radical groups for attracting individuals the population radicalized shows an exponential decay. Initial distribution $(0.9,0.1,0)$ and parameter values: $\alpha=0.1, \beta=0.1$, $\gamma=0.1, \delta=0.2$ and $p=0.5(\Omega=\Lambda=1 / 2<1)$.

\subsubsection{Case 2: $\Omega>1 \wedge \Lambda<1$}

In this case both $P_{1}$ and $P_{2}$ are equilibrium points. The condition $\Omega>1$ implies that $\lambda_{2}^{P_{1}}>0$, therefore $P_{1}$ now has an unstable direction (saddle point). 
As for $P_{2}$, the eigenvalues at that point are the following:

$$
\left\{\begin{array}{l}
\lambda_{1}^{P_{2}}=\frac{p \alpha \delta-\beta \gamma}{\beta}=\gamma\left(\frac{\Lambda}{\Omega}-1\right)<0 \\
\lambda_{2}^{P_{2}}=\delta-\beta=\delta(1-\Omega)<0
\end{array}\right.
$$

Note that if we start from case 1 and we increase $\Omega$, a forward bifurcation occurs in $\Omega=1\left[28\right.$ ] so $P_{1}$ loses its stability as the new stable equilibrium point is established:

$$
P_{2}=\left(\frac{1}{\Omega}, 0,1-\frac{1}{\Omega}\right) .
$$

This equilibrium point is the one that exists before the emergence of the radical group and is stable in both cases. This implies that the inclusion of some radicals will not modify the distribution, and the population will continue to be distributed between the susceptible and immunized categories in the same proportions as before, defined by $\Omega$. This situation corresponds to the case of societies with high cohesion levels $(\Omega>1)$, in which the strength of the radical group remains at low levels $(\Lambda<1)$. In particular, in the limit, $\Omega \gg 1$ the majority of the society will belong to the moderate group. The evolution of the populations for the same initial situation as in the previous case is shown in Fig. 3, where, the yellow and green curves will be approximately constant, given that $r(0) \ll 1$.

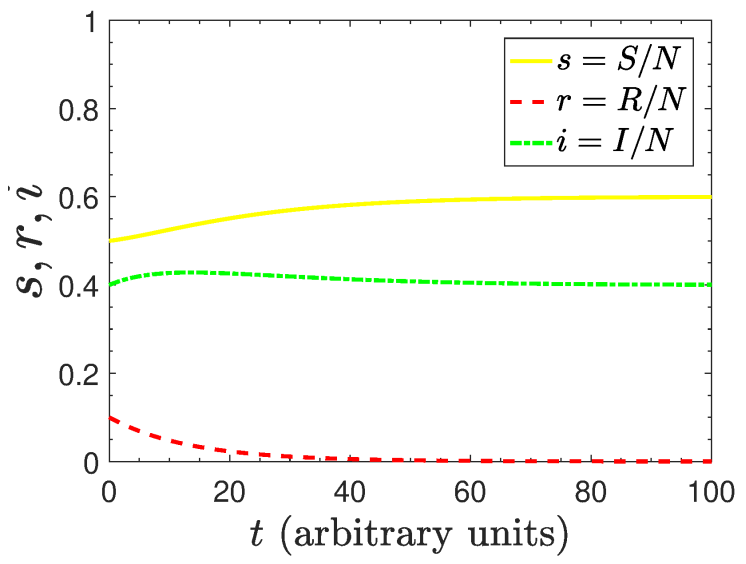

Figure 3: Coexistence of susceptible and moderate groups in a society with high cohesion levels and radical groups exhibiting weak strength. The initial condition is $(0.5,0.1,0.4)$ and the parameter values are $\alpha=0.1, \beta=1 / 6, \gamma=0.1, \delta=0.1$ and $p=0.5(\Omega=5 / 3>1$, $\Lambda=1 / 2<1)$.

\subsubsection{Case 3: $\Omega<1 \wedge \Lambda>1$}

As noted, the condition $\Omega<1$ implies that in the absence of radicals, the entire population will be susceptible. By introducing a small fraction of radicals, 
the dynamics becomes governed by the system (2), which will have $P_{1}$ and $P_{3}$ as equilibrium points. From Eq. 8 we observe that $\lambda_{1}^{P_{1}}>0$ and $\lambda_{2}^{P_{1}}<0$, therefore $P_{1}$ is a saddle point. The proof of the asymptotic stability of $P_{3}$, which is cumbersome due to the complex expression of the eigenvalues, can be analyzed with the signs of the trace and the determinant of the Jacobian matrix. Again, if we start from case 1 and increase $\Lambda$ a forward bifurcation occurs in $\Lambda=1$.

This case corresponds to radical groups that evolved towards more efficient organizations in at least one of the aspects of recruitment and retention and that are immersed in weakly cohesive societies. Consequently, the dynamics, shown in Fig. 4, reveals a sustained growth of such a group, whose proportion will constitute a non-negligible fraction of the total population in the asymptotic regime. In fact, in the $\Lambda \gg 1$ limit it is possible that large percentages of the population will end up adhering to the radical group. There are plenty of historical examples where this has happened, for example at the political level as a consequence of the emergence of charismatic radical leaders in societies undergoing serious periods of crisis 29 .

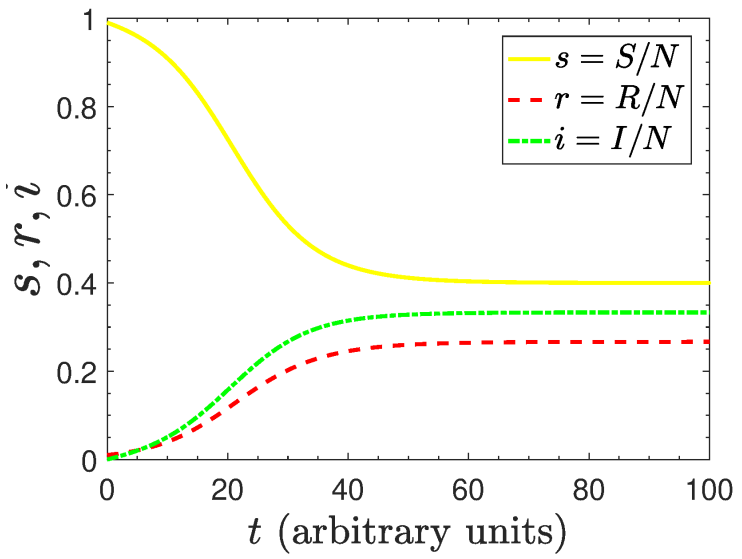

Figure 4: Strong radical groups in societies with low cohesion levels correspond to case 3. The values taken for the parameters are $\alpha=0.5, \beta=0.1, \gamma=0.1, \delta=0.2$ and $p=0.5$ $(\Omega=1 / 2<1, \Lambda=5 / 2>1)$. The initial distribution of populations is $(0.99,0.01,0)$.

We also note that despite the high social fragility $(\Omega<1)$, the asymptotic state can present an considerable number of moderates, a fact that did not occur prior to the appearance of the radical group. It is reasonable to suppose then that this occurs mainly as a response to radicalization, linked to the fraction $(1-p)$ and not so much due to the abandonment of the radical group nor to the intervention capacity of the immunized individuals.

\subsubsection{Case 4: $\Omega>\Lambda>1$}

Under these conditions the only equilibrium points are $P_{1}$ and $P_{2}$. The longterm behavior is identical to that of case 2 , since the only difference is that $P_{1}$ 


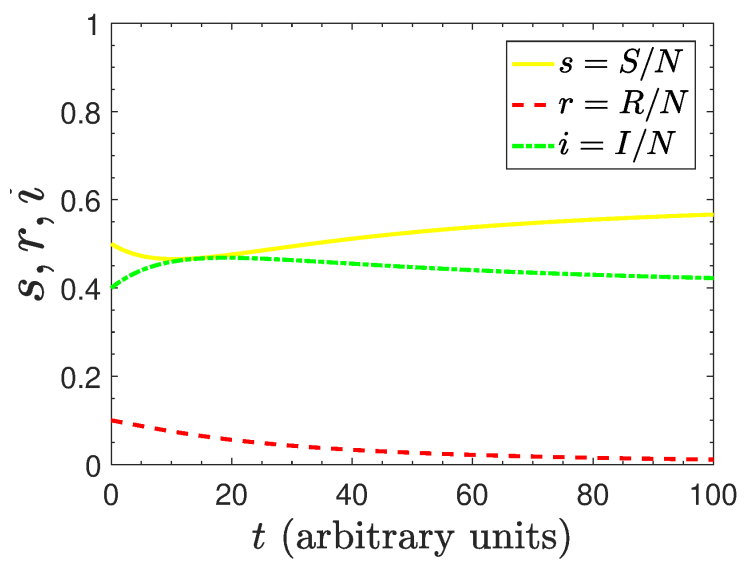

Figure 5: In societies with high cohesion levels the radical groups will remain in a marginal level regardless of their strength in case 4 . The values of the parameters are $\alpha=0.3, \beta=1 / 6$, $\gamma=0.1, \delta=0.1$ and $p=0.5(\Omega=5 / 3>\Lambda=3 / 2>1)$. The initial distribution of populations is $(0.5,0.1,0.4)$.

goes from being a saddle point to being unstable. Therefore, the equilibrium state coincides again with the state prior to the appearance of the radicals, whose long-term expression is again marginal. This occurs despite the fact that the radicals have increased their strength, a fact that manifests itself in two ways: on the one hand, in a longer dispersion time of that group; on the other hand, in a scenario of light growth at the beginning of the evolution, given that, if $\Lambda$ is large enough, $\Lambda s_{0}$ can be greater than 1 (see Eq. 9). This does not occur for the parameter values chosen in Fig. 5. but it was verified for other values. This case shows that in sufficiently cohesive societies, radical ideas will have little place regardless of their strength. As we will see below, this fact can be used to develop containment strategies.

\subsubsection{Case 5): $\Lambda>\Omega>1$}

We note, once again, that the coexistence of three equilibrium points, $P_{1}$ (unstable), $P_{2}$ (saddle point) and $P_{3}$ (asymptotically stable), implies that radicals will have an important expression. However, faced with a larger set of moderates than in case 3 , since the higher social cohesion ensures the functioning of the other immunization mechanisms. It is also possible to see that as increases $\Lambda$ for a $\Omega$ fixed, the number of susceptible individuals in the equilibrium state decreases, resulting in a polarized society between radical and immunized individuals. The evolution of the populations is shown in Fig. 6. 


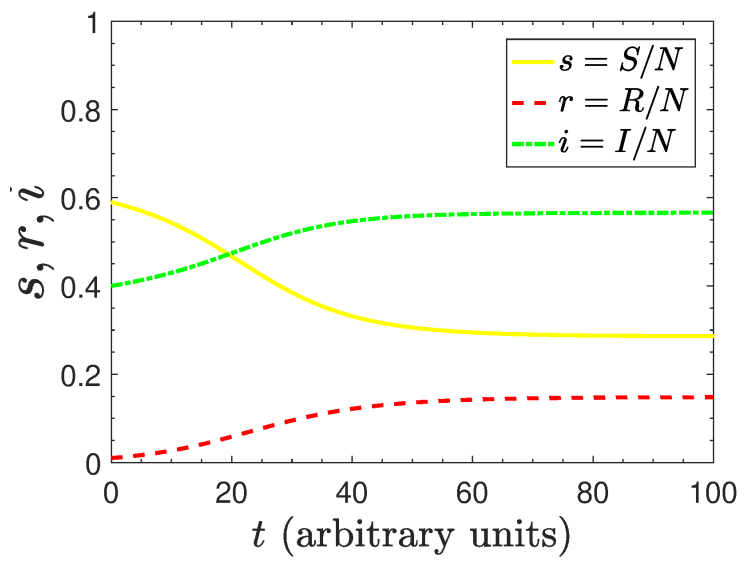

Figure 6: Case 5 corresponds to highly polarized societies. The values of the parameters are $\alpha=0.7, \beta=1 / 6, \gamma=0.1, \delta=0.1$ and $p=0.5(\Lambda=7 / 2>\Omega=5 / 3>1)$. The initial distribution of populations is $(0.59,0.01,0.4)$

\section{Modeling of systems with power-law memory: the Caputo deriva- tive}

It is important to note that when modeling some aspect of human societies using ordinary differential equations (such as Eq. 2), we are not taking the effects of memory into account. Although this a reasonable approximation in many cases of interest, it is to be expected that in situations such as the one studied in this work, the history of individuals and of society itself will play a non-negligible role.

Specifically, there is a broad evidence that the processes that involve the use of memory in humans follow power laws [30. In particular, various experiments have shown that the decrease in efficiency achieved in memorization-based tasks decreases as $t^{-\epsilon}$, where $0<\epsilon<1$ 31. Reciprocally, learning (measured in terms of the decrease in reaction times to certain stimuli) also follows a power law in the number of attempts [32. Usually this behavior of memory in mammals is explained as a consequence of the corresponding power-law memory that neurons present, as well as a good part of the rest of cells and tissues due to to their viscoelastic character.

As we have already commented, the inclusion of memory in the evolution equations can be implemented by using fractional calculus. To introduce the fractional derivatives, we start from the Cauchy formula for iterated integrals of order $n \in N$ :

$$
I_{0}^{n} f=\frac{1}{(n-1) !} \int_{0}^{t}\left(t-t^{\prime}\right)^{n-1} f\left(t^{\prime}\right) d t^{\prime}
$$

and extend it to an arbitrary positive index $b$, using Euler's $\Gamma$ function as an analytic continuation of the factorial over the real numbers. Thus, we obtain 


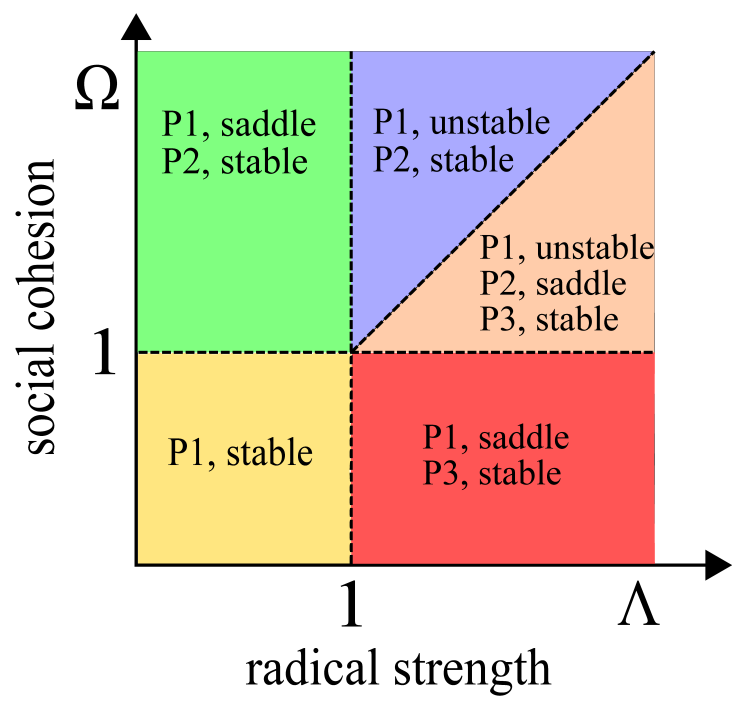

Figure 7: Relevant regions in the parameter space $(\Lambda, \Omega)$, defined as a function of the present equilibrium points and their stability.

the Riemann-Liouville fractional integral:

$$
I_{0}^{b} f=\frac{1}{\Gamma(b)} \int_{0}^{t}\left(t-t^{\prime}\right)^{b-1} f\left(t^{\prime}\right) d t^{\prime}
$$

Among the different definitions of fractional derivatives, in this work we will rely on the Caputo derivative, which, for an arbitrary order of differentiation $a>0$, is defined as 33

$$
{ }^{C} D_{0}^{a} f=I^{n-a} D^{n} f=\frac{1}{\Gamma(n-a)} \int_{0}^{t}\left(t-t^{\prime}\right)^{n-a-1} D^{n} f\left(t^{\prime}\right) d t^{\prime}
$$

where $n$ is the first integer greater than $a$, and $D^{n}$ is the usual differentiation operator of order $n$.

The first advantage that the use of this derivative presents with respect to other definitions is that initial value problems with Caputo derivative are initialized by integer-order derivatives [34]:

$$
\left\{\begin{array}{l}
{ }^{C} D_{0}^{a} x(t)=f(t, x) \\
x(0)=x_{0}, x^{\prime}(0)=x_{0}^{\prime}, \ldots, x^{(n-1)}(0)=x_{0}^{n-1},
\end{array}\right.
$$

unlike what occurs when employing other definitions (such as the RiemannLiouville derivative, which requires the knowledge of fractional derivatives as initial values).

Another important advantage of the Caputo derivative is related to its action on constant functions. Since the derivatives of any order of a constant function 
$f$ are zero for all times, Eq. (14) implies that

$$
{ }^{C} D_{0}^{a} f=0,
$$

just like in the standard case. As a consequence, if we consider a system of ordinary differential equations (such as Eq. (2)), and substitute all derivatives by fractional derivatives, the equilibrium points of the fractional system will be the same as those of the original system. It is important to emphasize that this does not occur with other definitions of fractional derivative [34. garr In order to understand how the Caputo derivative can play an important role in the modeling of systems with memory, let us consider a first order differential equation of the type:

$$
D^{1} x(t)=f(x(t))
$$

where $D^{1}$ is the common derivative. Since the rate of change of the unknown function $x(t)$ depends only on the value of $f$ at the present time, the system described by Eq. (17) has no memory. To include memory in the system, we will assume that the rate of change $D^{1} x(t)$ is no longer equal to the value of $f$ at that point, but rather depends on the previous values of $f$ through its convolution with a function $\varphi$, called the memory kernel:

$$
D^{1} x(t)=\int_{0}^{t} \varphi\left(t-t^{\prime}\right) f\left(x\left(t^{\prime}\right)\right) d t^{\prime}
$$

Different choices of $\varphi$ are associated with different types of memory. For example, if we take as kernel the Dirac delta distribution, we obtain Eq. (17), which corresponds to the Markovian case. On the opposite hand, choosing the derivative of the previous distribution as kernel, known as the Heaviside step function, implies that the system has infinite memory, since it assigns equal weight to all the previous points.

Since our interest is to model human societies, according to the empirical evidence already discussed, it is reasonable to choose $\varphi$ functions that decay as power laws. In particular

$$
\varphi\left(t-t^{\prime}\right)=\frac{\left(t-t^{\prime}\right)^{a-2}}{\Gamma(a-1)} .
$$

By replacing this kernel in Eq. 18, we obtain the following:

$$
D^{1} x(t)=\frac{1}{\Gamma(a-1)} \int_{0}^{t}\left(t-t^{\prime}\right)^{a-2} f\left(x\left(t^{\prime}\right)\right) d t^{\prime} .
$$

It should be noted, however, that by virtue of the definition $(13)$, the r.h.s. of Eq. 20 is nothing other than the Riemmann-Liouville fractional integral of order $a-1$ :

$$
\frac{1}{\Gamma(a-1)} \int_{0}^{t}\left(t-t^{\prime}\right)^{a-2} f\left(x\left(t^{\prime}\right)\right) d t^{\prime}=I_{0}^{a-1} f(x(t)),
$$


therefore, Eq. 18 becomes

$$
D^{1} x(t)=I_{0}^{a-1} f(x(t)) .
$$

Applying the $I_{0}^{1-a}$ operator to both sides of the above equation, we obtain the following

$$
I_{0}^{1-a} D^{1} x(t)=I_{0}^{1-a} I_{0}^{a-1} f(x(t)) .
$$

Finally, note that the composition of the operators on the right-side term is equivalent to the identity operator, while the left-side term is, by virtue of the definition [33, the Caputo derivative of order $a(0<a \leq 1)$, so we obtain the following:

$$
{ }^{C} D_{0}^{a} x(t)=f(x(t)) .
$$

In summary, if we assume that the rate of change of the function we seek, $x(t)$, does not depend on $f$ at the point, but rather on $f$ weighed by a memory kernel that decays as a power law, then the dynamics of the system will no longer be governed by an ordinary differential equation, but by one of fractional order less than 1 (equivalent to an ordinary integral-differential equation). It is also possible to see that the degree of memory of the system is controlled by the order of the derivative: as approaches the value 1, the system has less memory [35, and in the extreme case of $a=1$ we return to the Markovian model, which is described correctly by an ordinary differential equation.

Applying these ideas to the model described above, we obtain the following fractional system of order $a$ :

$$
\left\{\begin{array}{l}
{ }^{C} D_{0}^{a} s=-\alpha s r-\beta s i+\delta i \\
{ }^{C} D_{0}^{a} r=p \alpha s r-\gamma r \\
{ }^{C} D_{0}^{a} i=\gamma r+\beta s i-\delta i+(1-p) \alpha s r
\end{array}\right.
$$

which will be analyzed in the next subsection.

\section{Memory effects in the radicalization process}

As we have already mentioned, the equilibrium points of the system (25) coincide with those of the analyzed Markovian case. The asymptotic stability in the fractional case is assured if the two eigenvalues of the Jacobian matrix of the system, evaluated at that point, satisfy the condition $\left|\operatorname{Arg}\left(\lambda_{i}\right)\right|>a \pi / 2[36$. This result elegantly generalizes the well-known result for ordinary systems [37. In particular, if the eigenvalues are real, their argument can only be 0 (positive real) or $\pi$ (negative real). Since we work under the restriction $0<a \leq 1$, and by virtue of Eqs. (8) and (10), we conclude that $P_{1}$ and $P_{2}$ (and hence also $P_{3}$ ) will be asymptotically stable in the same regions as in the ordinary problem. This implies that the inclusion of memory does not produce any modification regarding the final distribution of the populations of the system. However, 

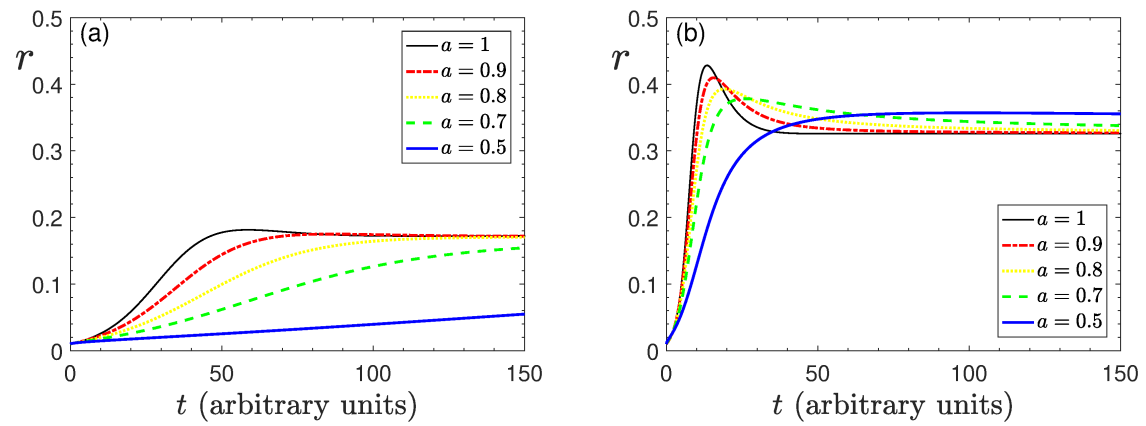

Figure 8: Evolution of the radicalized for case 3, considering different levels of memory and different strengths of the radical group. Parameter values are such that: a) $\Omega=1 / 2, \Lambda=9 / 5$; b) $\Omega=1 / 2, \Lambda=6$. The numerical methods employed are described in Ref. [34.

we will see that it does have significant effects on the evolution towards the asymptotic state.

Figure 8 shows the evolution of the radicalized individuals for two situations corresponding to case 3 , but at different limits of strength of the radical group. In the first graph (relatively strong radical group) we observe that the Markovian approximation underestimates the duration of the transient regime, the longer the times required to reach the steady state the more memory is included in the system (the time scales required can be very different). On the other hand, the second graph shows that, in the presence of a very strong radical group, models with little memory predict the existence of radicalization peaks before the final distribution is reached. When more memory is included, this behavior tends to disappear and in the infinite memory limit the radicals grow monotonically until reaching their equilibrium value. Although more memory implies, once again, that the process is more gradual (peaks occur at later times), the difference is less noticeable than in the previous case.

One of the main uses of epidemiological models is to analyze the effect of possible interventions and to provide useful information for designing epidemic control strategies [2]. In the context of the present model, the question arises as to what measures can be employed to combat radicalization and what are their effects. Given that the behavior of the system in the long run is governed by the $\Lambda$ and $\Omega$ parameters, and that radicals will have a marginal expression whenever $\Omega>\Lambda$, , appropriate measures should tend to decrease $\Lambda$ and/or increase $\Omega$. However, not all the original parameters of the model are externally controllable. The parameter $\alpha$ will depend on the level of militancy of the radicals, $\gamma$ on the level of contentment provided by belonging to such a group; and $p$ on the efficiency of their convincing strategies as well as on cultural factors. On the other hand, $\delta$ is a measure of the probability that a moderate individual will become susceptible, therefore it will depend on random personal factors and others linked to social welfare, which can only be modified in the mediumto-long term 38. These hypotheses leave as the main measure the application 
of policies that encourage positive social interaction (increasing $\beta$ ), trying to appease the radical impulse by depriving it of susceptible individuals.
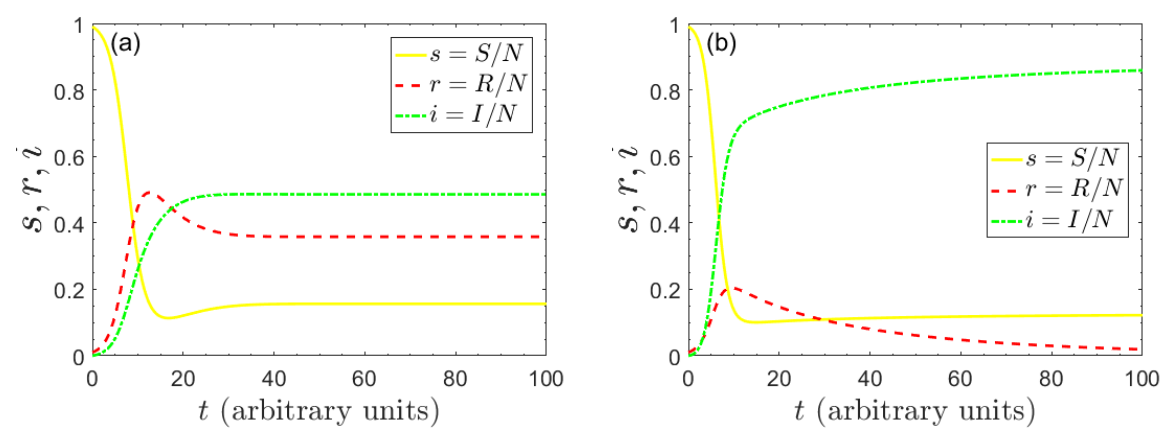

Figure 9: Evolution of the populations in the presence of a strong radical group (Markovian case), without intervention (panel (a), $\Omega=0.05, \Lambda=6.4$ ); and with a strong intervention (panel (b), $\Omega=8, \Lambda=6.4)$. The initial distribution is $(0.99,0.01,0)$.

As an example, let us consider again the situation corresponding to case 3 , for large values of $\Lambda$. According to the previous analysis, the population will start at the susceptible state, and if the system evolves without any intervention, there will be a significant fraction of radicals in the equilibrium state (see Fig. 9a). Suppose that, to avoid this, strong intervention strategies are implemented on the susceptible population. If $\Omega$ manages to surpass $\Lambda$ (case 5 ), the evolution of the populations predicted by the Markovian model will be similar to that shown in Fig. 9 $\mathrm{b}$. The temporal evolution in this case exhibits a maximum, but with smaller amplitude than in the previous case, concurrent with an increase in immunized individuals. After overcoming this maximum, the radical fraction vanishes in the long term.

The effects of this policy in societies with different levels of memory are shown in Fig. 10. We have verified that the increase in positive interaction with the susceptible population has the effect described in the above paragraph independently of the memory level. However, the characteristics of the remaining radicalization peak, as well as of the subsequent evolution towards the asymptotic state, strongly depend on the parameter $a$. Note that, although the time of occurrence of the peak is essentially the same in all cases, the more memory is included in the system, the smaller the amplitude of the peak and the longer the time required to reach an equilibrium. For practical purposes, we conclude that the application of this policy:

- It does not manage to delay the advance of radicalization, in the sense that the maximum proportion of radicals is reached at the same time as if the policy had not been implemented. Additionally, that time is essentially independent of the memory of the system.

- It is successful in reducing the maximum levels of radicalizaion, for all memory levels. 

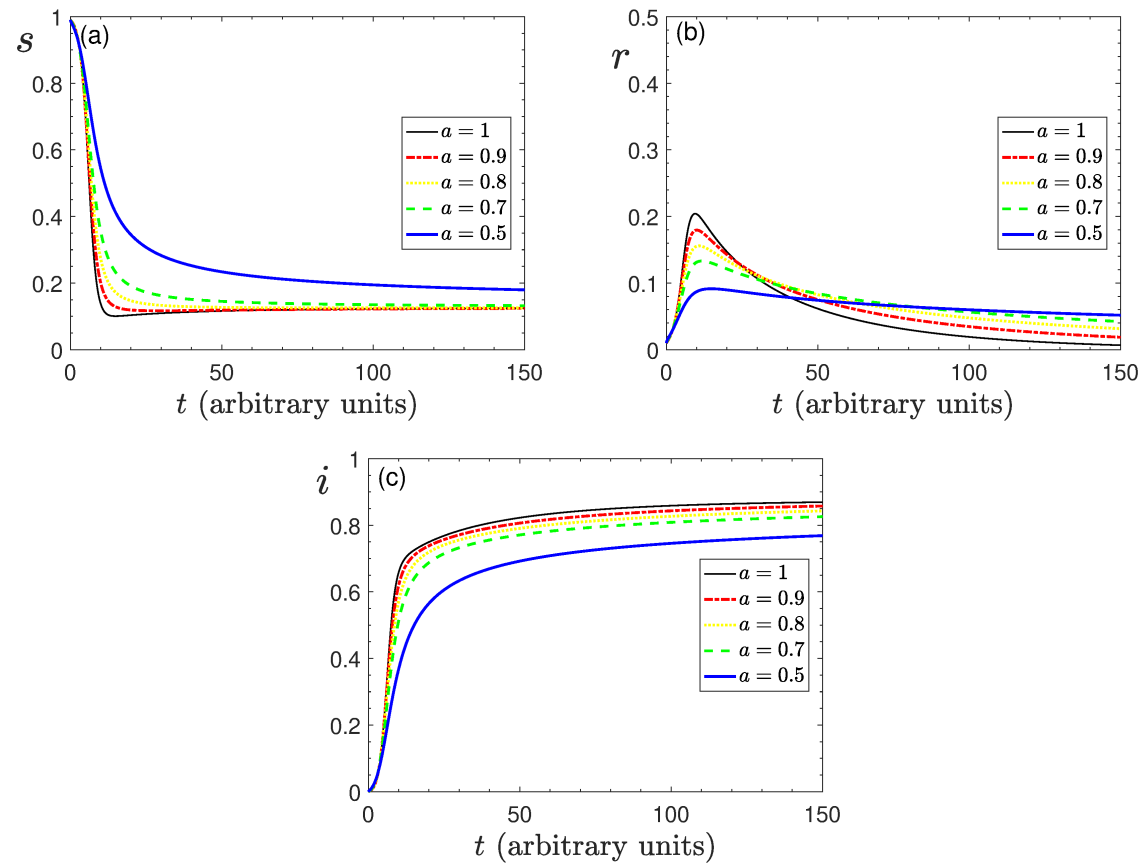

Figure 10: Evolution of populations; susceptibles (a), radicalized (b) and inmunized (c), once the interaction with susceptibles has been increased for different levels of memory. The values of the parameters are: $\alpha=0.8, \beta=0.8, \gamma=0.1, \delta=0.1$ and $p=0.8(\Omega=8, \Lambda=6.4)$. The initial population distribution is $(0.99,0.01,0)$.

- It is successful in the long term, reducing radicalization to negligible values.

- It is more successful in overcoming radicalization in societies with shortrange memory. The more memory is included in the modeling, the longer the coexistence with the radical group.

\section{Conclusion}

In this work we have proposed a simple compartmental model, inspired by epidemiological models, to describe the dynamics of radical ideas in a society with memory. The model reasonably predicts under what conditions the radical group remains in marginal expressions, achieves a stable presence, or eventually manages to impose itself as the main expression.

The model depends strongly on two parameters. One of them, $\Lambda$, represents the number of susceptible individuals that each radical persuades into joining the group while professing that ideology, thus representing a measure of the strength of that group. The other quantity, $\Omega$, plays the same role as $\Lambda$, but for 
the immunized group, and ensures the disappearance of radicals whenever its value exceeds $\Lambda$. This parameter can be interpreted from a sociological point of view, noting that the specific literature agrees that radicalization arises less frequently in highly cohesive societies. High cohesion implies, on the one hand, a reasonable level of social welfare related to economic aspects, public health, low level of inequality and respect for minorities (linked in our model to the parameter $\delta$ ); but also to the fact that all groups that make up society feel duly integrated, the existence of shared values and a strong interconnection and solidarity among its members (aspects related to $\beta$ ). Given that $\Omega$ is constructed based on these two parameters, we consider that it can represent a reasonable measure of social cohesion.

The model also highlights the importance of intervention strategies on vulnerable populations as a tool to control radicalization. The difficulties faced by deradicalization programs and intelligence services in combating violent radicalization suggest the need for a paradigm shift, in which greater importance is given to strategies aimed at preventing radicalization in its early stages 39, 40. Examples include interventions in educational settings aimed at youth and adolescents 22, 41, or roundtable discussions to include minority representatives 42. These types of interventions aim at increasing the interaction between immunized and susceptible individuals, so they can be associated with an increase of $\beta$ (and hence of $\Omega$ ). In the context of this model, we have seen that these measures, although they do not stop the advance of radicalization, they flatten the curve and manage to maintain it at negligible levels in the long term.

The effects of including memory in the modeling of the system plays a significant role. The level or degree of memory is a characteristic of societies over which there is no immediate control. However, it is interesting to analyze how the result of the intervention policies may be different, depending on the degree of memory that a particular society presents. We observe that at higher levels of memory, processes occur less abruptly, and it takes longer to reach a state of equilibrium. In terms of combating radicalization through interaction with susceptible individuals, the model predicts that societies with more memory will have lower values of transient radicalization, but a longer time of coexistence with the radical group before its disappearance.

\section{Acknowledgements}

This work was partially supported by Comisión Académica de Posgrado (CAP), Agencia Nacional de Investigación e Innovación (ANII) and Programa de Desarrollo de las las Ciencias Básicas (PEDECIBA) (Uruguay).

\section{References}

[1] W. O. Kermack, A. G. McKendrick, Contributions to the mathematical theory of epidemics. ii.- the problem of endemicity, Proceedings of the Royal Society of London. Series A, containing papers of a mathematical and physical character 138 (834) (1932) 55-83. 
[2] F. Brauer, C. Castillo-Chavez, C. Castillo-Chavez, Mathematical models in population biology and epidemiology, Vol. 2, Springer, 2012.

[3] W. Goffman, V. Newill, Generalization of epidemic theory, Nature 204 (4955) (1964) 225-228.

[4] L. M. Bettencourt, A. Cintrón-Arias, D. I. Kaiser, C. Castillo-Chávez, The power of a good idea: Quantitative modeling of the spread of ideas from epidemiological models, Physica A: Statistical Mechanics and its Applications 364 (2006) 513-536.

[5] D. M. Abrams, S. H. Strogatz, Modelling the dynamics of language death, Nature 424 (6951) (2003) 900-900.

[6] F. Jin, E. Dougherty, P. Saraf, Y. Cao, N. Ramakrishnan, Proceedings of the 7th workshop on social network mining and analysis.

[7] J. Bissell, C. Caiado, M. Goldstein, B. Straughan, Compartmental modelling of social dynamics with generalised peer incidence, Mathematical Models and Methods in Applied Sciences 24 (04) (2014) 719-750.

[8] P. B. Stares, M. Yacoubian, Terrorism as a disease: An epidemiological model for countering islamist extremism, Mathew B Ridgeway Centre for international Security Studies. Pittsburgh. United States.

[9] S. Galam, M. Javarone, Plos one 11, e0155407 (2016).

[10] C. C. McCluskey, M. Santoprete, A bare-bones mathematical model of radicalization, Journal of Dynamics \& Games 5 (3) (2018) 243.

[11] M. B. Short, S. G. McCalla, M. R. D’Orsogna, Modelling radicalization: How small violent fringe sects develop into large indoctrinated societies, Royal Society open science 4 (8) (2017) 170678.

[12] S. Nizamani, N. Memon, S. Galam, From public outrage to the burst of public violence: An epidemic-like model, Physica A: statistical mechanics and its applications 416 (2014) 620-630.

[13] H. T. Banks, C. Castillo-Chavez, Bioterrorism: mathematical modeling applications in homeland security, SIAM, 2003.

[14] R. Hilfer, Applications of fractional calculus in physics, World scientific, 2000 .

[15] H. Sun, Y. Zhang, D. Baleanu, W. Chen, Y. Chen, A new collection of real world applications of fractional calculus in science and engineering, Communications in Nonlinear Science and Numerical Simulation 64 (2018) $213-231$.

[16] M. Duarte Ortigueira, J. Tenreiro Machado, Fractional derivatives: The perspective of system theory, Mathematics 7 (2) (2019) 150. 
[17] M. Hafez, C. Mullins, The radicalization puzzle: A theoretical synthesis of empirical approaches to homegrown extremism, Studies in Conflict \& Terrorism 38 (11) (2015) 958-975.

[18] C. Kurzman, The Missing Martyrs: Why are There So Few Muslim Terrorists?, Oxford University Press, 2018.

[19] J. G. Horgan, Walking away from terrorism: Accounts of disengagement from radical and extremist movements, Routledge, 2009.

[20] H. Heesterbeek, The law of mass-action in epidemiology: a historical perspective, Ecological paradigms lost: routes of theory change (2005) 81-104.

[21] M. Sedgwick, Inspiration and the origins of global waves of terrorism, Studies in Conflict \& Terrorism 30 (2) (2007) 97-112.

[22] E. Tsintsadze-Maass, R. W. Maass, Groupthink and terrorist radicalization, Terrorism and Political Violence 26 (5) (2014) 735-758.

[23] M. Hewstone, E. Cairns, Social psychology and intergroup conflict.

[24] D. Della Porta, Social movements, political violence, and the state: A comparative analysis of Italy and Germany, Cambridge University Press, 2006.

[25] J. Lalich, Bounded choice: True believers and charismatic cults, Univ of California Press, 2004.

[26] I. Kawachi, L. Berkman, et al., Social cohesion, social capital, and health, Social epidemiology 174 (7).

[27] H. E. Alcalá, M. Z. Sharif, G. Samari, Social determinants of health, violent radicalization, and terrorism: a public health perspective, Health Equity 1 (1) (2017) 87-95.

[28] A. B. Gumel, Causes of backward bifurcations in some epidemiological models, Journal of Mathematical Analysis and Applications 395 (1) (2012) $355-365$.

[29] T. S. Pappas, Political leadership and the emergence of radical mass movements in democracy, Comparative Political Studies 41 (8) (2008) 11171140 .

[30] M. Edelman, Evolution of systems with power-law memory: Do we have to die?(dedicated to the memory of valentin afraimovich), in: Demography of Population Health, Aging and Health Expenditures, Springer, 2020, pp. 65-85.

[31] M. J. Kahana, Foundations of human memory, OUP USA, 2012. 
[32] J. R. Anderson, Learning and memory: An integrated approach, John Wiley \& Sons Inc, 2000.

[33] M. Caputo, Linear models of dissipation whose q is almost frequency independent-ii, Geophysical Journal International 13 (5) (1967) 529-539.

[34] R. Garrappa, Numerical solution of fractional differential equations: A survey and a software tutorial, Mathematics 6 (2) (2018) 16.

[35] M. Du, Z. Wang, H. Hu, Measuring memory with the order of fractional derivative, Scientific reports 3 (1) (2013) 1-3.

[36] D. Matignon, Stability results for fractional differential equations with applications to control processing, in: Computational engineering in systems applications, Vol. 2, Lille, France, 1996, pp. 963-968.

[37] S. H. Strogatz, Nonlinear dynamics and chaos with student solutions manual: With applications to physics, biology, chemistry, and engineering, CRC press, 2018.

[38] Y. Berman, D. Phillips, Indicators for social cohesion, Paper submitted to the European Network on Indicators of Social Quality of the European Foundation on Social Quality, Amsterdam.

[39] M. Grossman, M. Peucker, D. Smith, H. D. AO, Stocktake research project, Melbourne, VIC, Australia: Community Resilience Unit, Department of Premier and Cabinet.

[40] C. Rousseau, G. Hassan, Y. Oulhote, And if there were another way out? questioning the prevalent radicalization models, Canadian Journal of Public Health 108 (5) (2017) e633-e635.

[41] K. S. Bhui, M. H. Hicks, M. Lashley, E. Jones, A public health approach to understanding and preventing violent radicalization, BMC medicine 10 (1) (2012) 1-8.

[42] E. Keeble, 10. the cross-cultural roundtable on security as a response to radicalization: Personal experiences and academic refl ections, in: Religious Radicalization and Securitization in Canada and Beyond, University of Toronto Press, 2018, pp. 259-284. 